
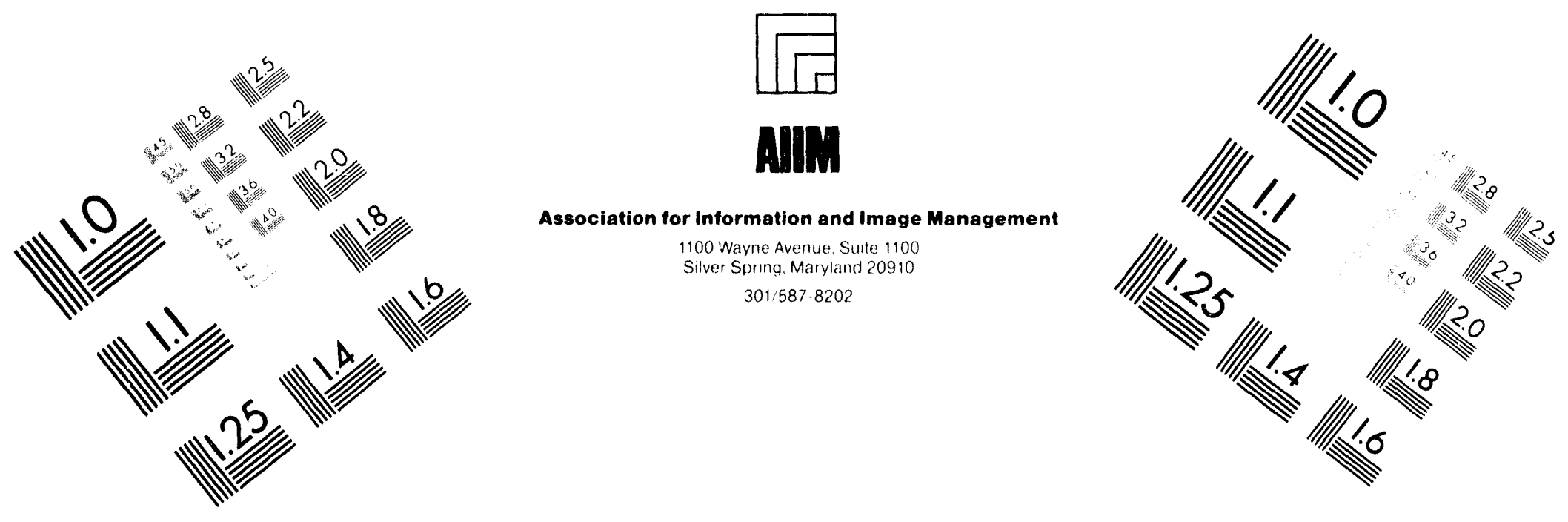

\title{
Centimeter
}

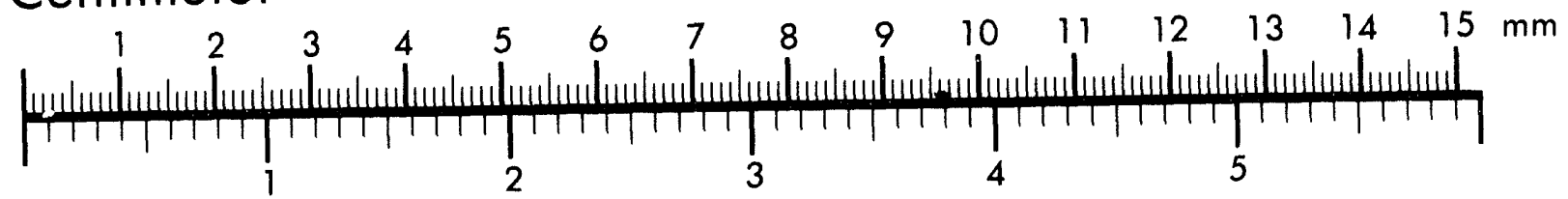
Inches
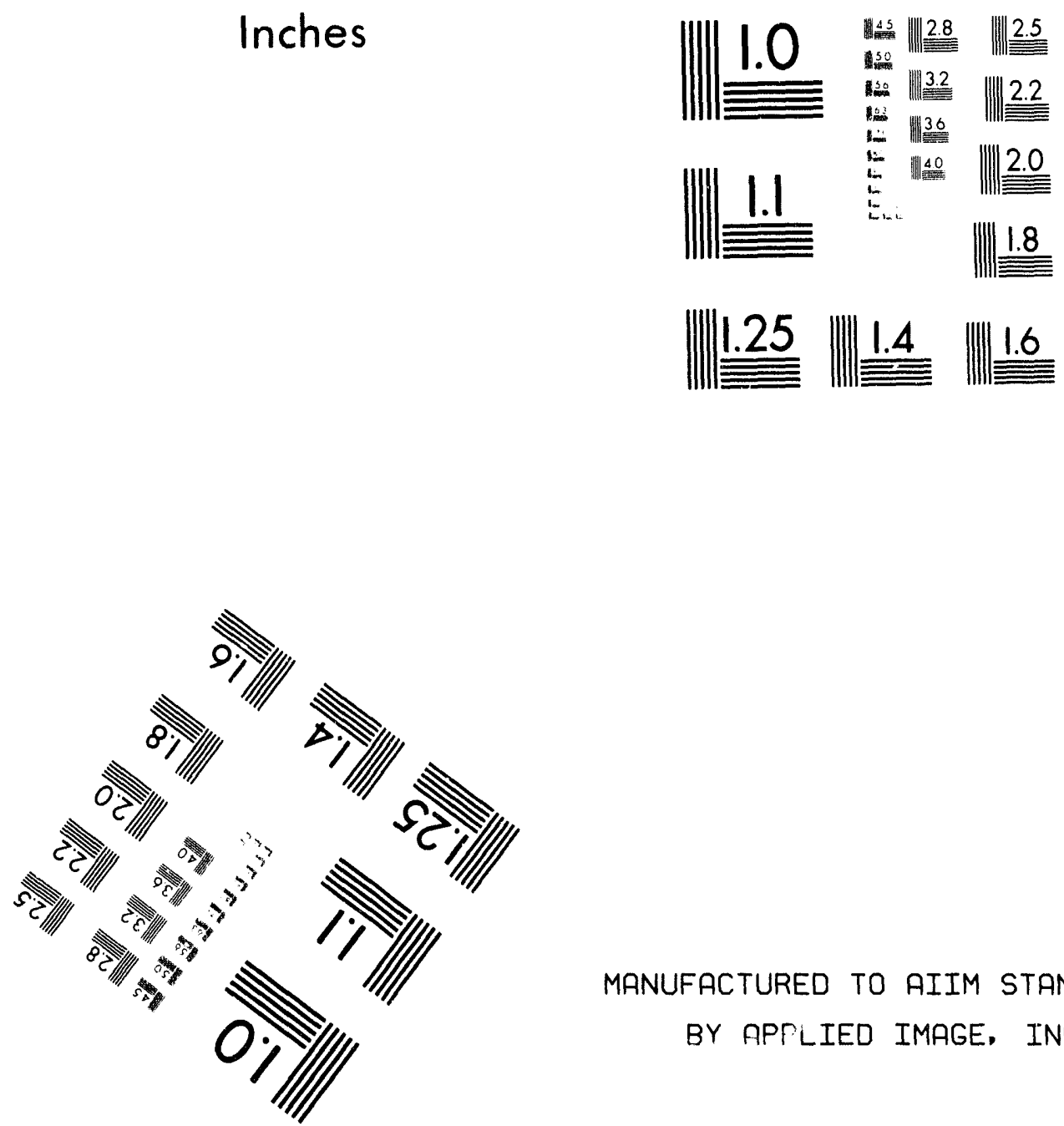

MANUFACTURED TO AIIM STANDARDS

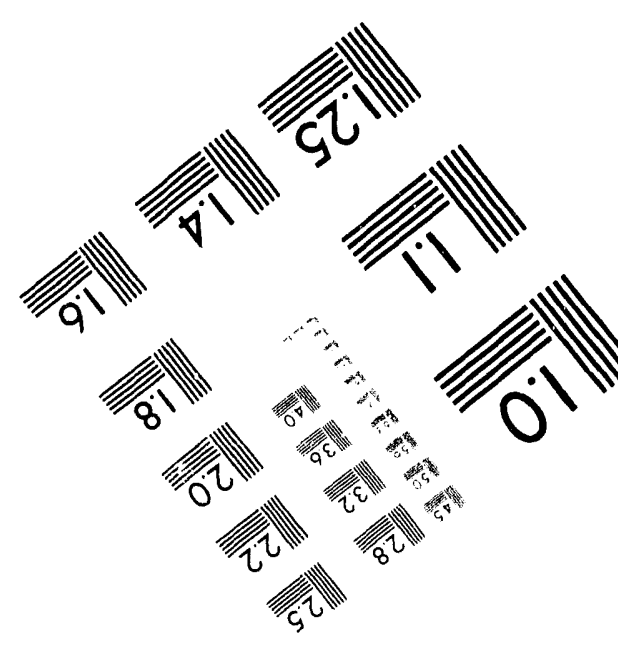



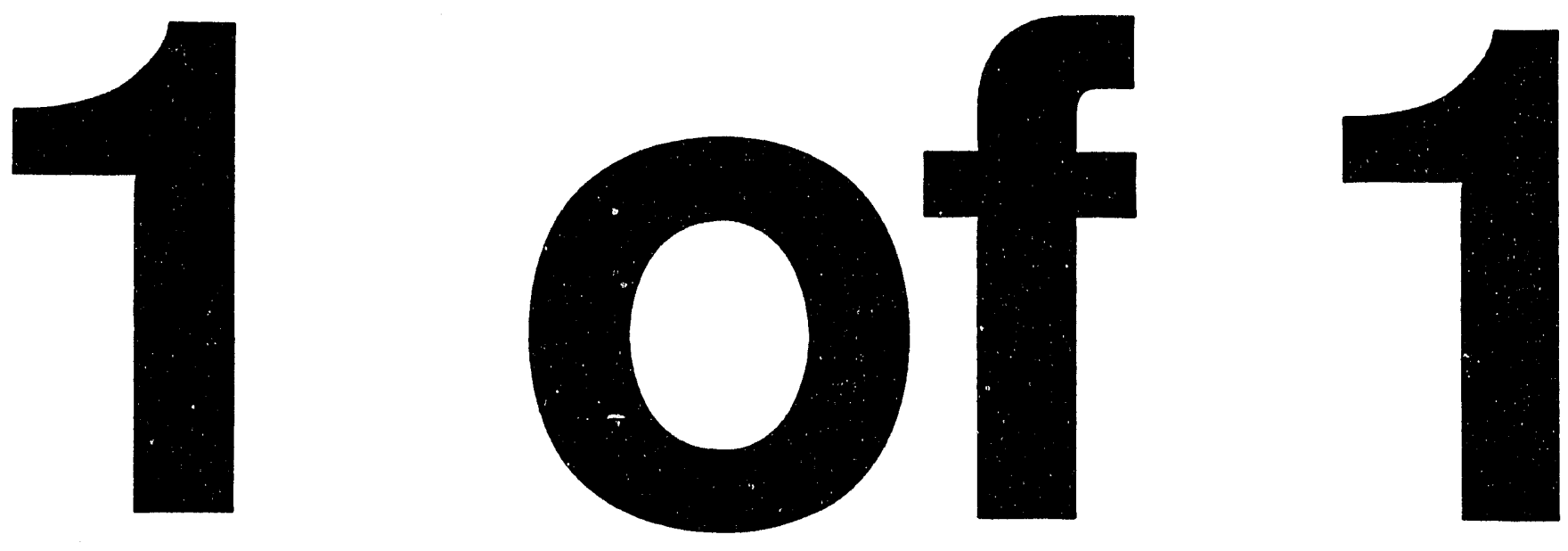

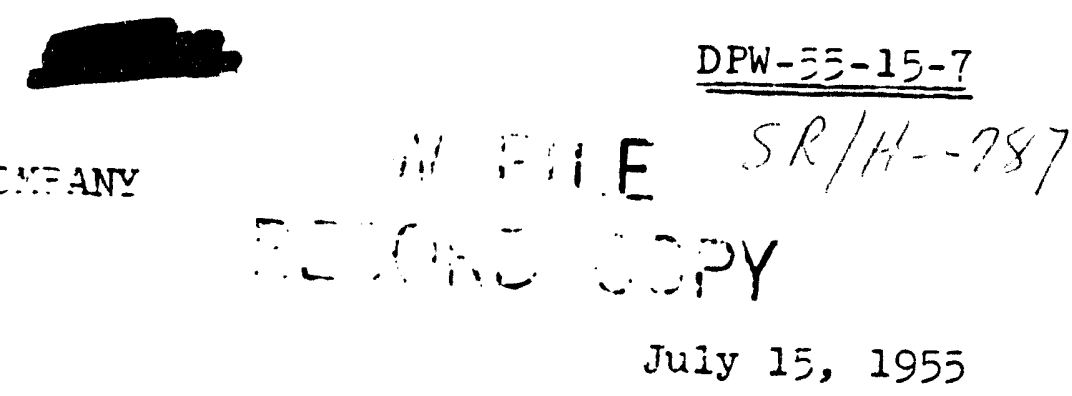

\section{DIS-R IBUTION}

FI - D. F. Babcock

岸 - L. C. Evans

$\# 3$ - B. H. Mackey

$\frac{u^{4}}{\pi}-\mathrm{S}$. A. McNeight

$\overline{\bar{\pi}} \bar{j}$ - V. R. Thayer

- I. C. Woodhouse

$=7$ - Hood Worthingtor:

$=3$ - "W" F1le

$\# 9-I$ - M. H. Wahl - SPL

FIE-C.W. J. Wende - SRI

$\div 3$ - "ST" File

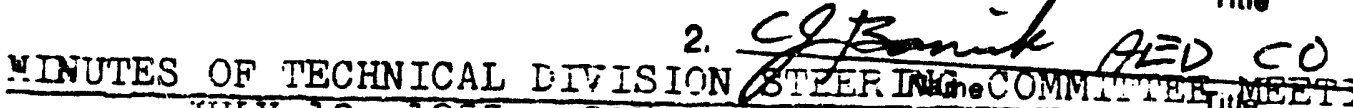
Classification Cancelled/Chand.

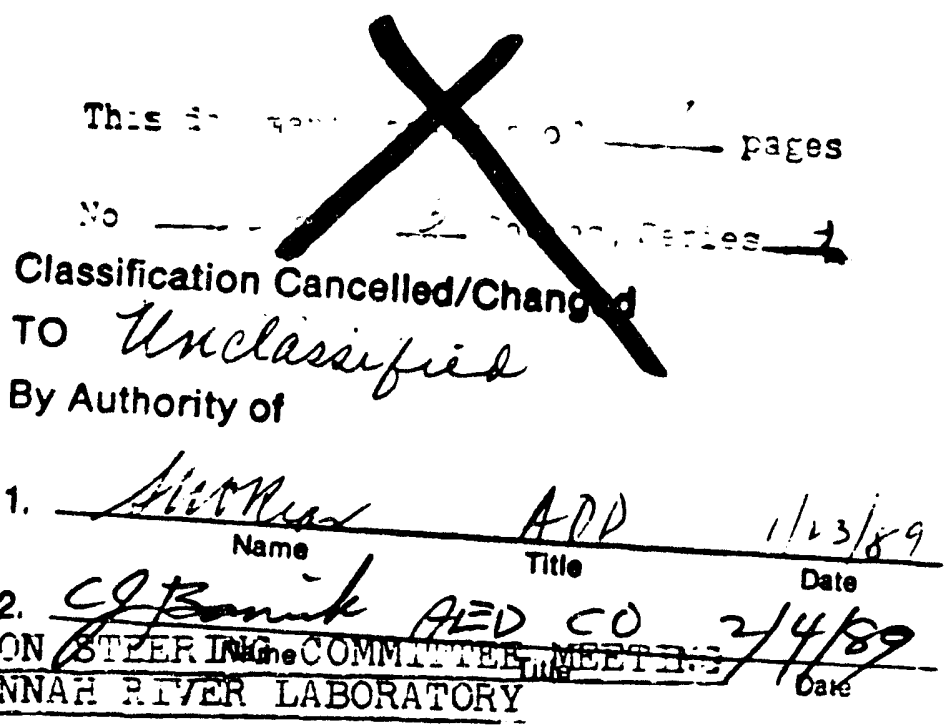

Committe Members Present

DOES NOT CONTA'.

D. F. Babcock:

J. W. Croach

Gerhard Diessauer

L. C. Evans

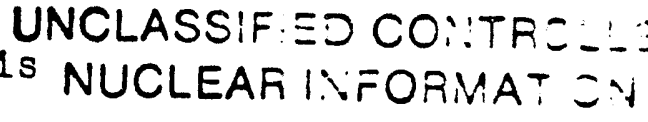

M. H. Wahl

C. W. J. Wanding

Locd wortbotnetion

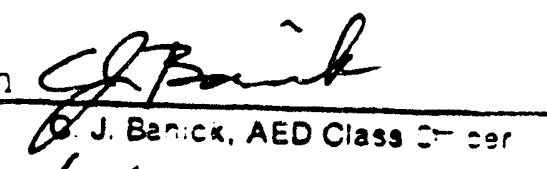

APFOOVALS

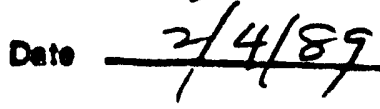

The following studies were approved for the programs outined in the appendixes:

St lidy

Nc.

$85 \mathrm{Cl}$ Non-destructive TES:1rg

8562

8563 Present Componer.ts

85c3 New Fuel Element Fabricatior.

8504 Protective Coatings for New

850 Fuel Elements

8503

8514

Corrosion

Instrument Deveiop=ent - 300 Area

Instrument Developrent - 2CO Area

8515

8524
Instrument Developrent - 100 Area New IM Elements
$\operatorname{Man}$

\begin{tabular}{|c|c|c|}
\hline Months & From & $=0$ \\
\hline $\begin{array}{l}0 \\
6\end{array}$ & $\begin{array}{l}7 / 1 / 55 \\
7 / 1 / 55\end{array}$ & $\begin{array}{l}c / 30 / 55 \\
\subseteq / 30 / 55\end{array}$ \\
\hline $\begin{array}{l}38 \\
39\end{array}$ & $\begin{array}{l}7 / 1 / 55 \\
7 / 1 / 55\end{array}$ & $\begin{array}{l}c / 30 / 55 \\
c / 30 / 55\end{array}$ \\
\hline $\begin{array}{l}13 \\
27\end{array}$ & $\begin{array}{l}7 / 1 / 55 \\
7 / 1 / 55\end{array}$ & $\begin{array}{l}E / 30 / 55 \\
E / 30 / 55\end{array}$ \\
\hline 42 & $7 / 1 / 55$ & $9 / 30 / 55$ \\
\hline 15 & $7 / 1 / 55$ & $9 / 30 / 55$ \\
\hline 18 & $7 / 1 / 55$ & $\begin{array}{l}9.30 / 55 \\
\text { Fage } 1\end{array}$ \\
\hline
\end{tabular}

DERMBBUTION D: THIO DOCUMENT IS OMLIMITEO 
INFORMATION AND AGREEMENTS REACHED

\section{Safety}

The Laboratory's miror infury frequency was 0.31 for June, approximately the same as for Hay. An analysis indicates that a major factor in June minor in iries was fallures to follow established procedures. This point is being emphasized.

More rigorous procedunss are being developed to ald in assuring that exposures to radisactivity are at a minimum.

2. The Laboratory had thr- scurity violations in June, a decrease from four in May. The AEC recently completed the Ir annual security survey of the Laboratory facilities and found everything in good order.

3. The work capacity of the high level caves is exceeded by the amount of work requested. The Laboratory is taking a number of immediate steps to increase the efflclency of cave work, and a survey is being made to establish if and what additional facilities will be needed in the future.

4. The Laboratory will participate in work directed toward the development of hollow slug fuel elements. The responsibilities and the extent of participation in this work are yet to be resolved.

5. The Laboratory instrument development group is making arrangements for suitable vendors to assist in the development and mechanization of various instruments. This presents patent problems both with respect to divulging information to these vendors and with respect to the type of patent acreements acceptable to trese vendors. I. C. Evans will work with the Laboratory to establish a simple procedure for coping with these patent considerations.

6. Several of the Ingots of slightly enriched uranium performed poorly during rolling. The Laboratory has carefully examined the plates produced from these ingots and has found no evidence that the plates are unsuitable for irradiation.

7. Tentatively, the next steering Committee Meeting w1ll be at the Laboratory on August 9 .

8. Attached for information are:

$$
\begin{aligned}
& \text { Append1x A - Pirancial Status } \\
& \text { Append1x B - File Materials Program } \\
& \text { Append1x C - Instrument Development Program } \\
& \text { Appendix D - Technical Division Study Status }
\end{aligned}
$$

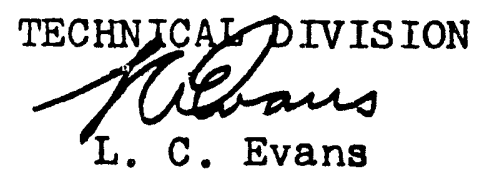




\section{APPENDIX A}

\section{FINANSIAL STATUS}

Technical Division expenditures for FY-1955 through May were about $\$ 150,000$, or 2\% under the Financial Plan. Although June costs w1ll be inflated by rather heavy year-end accruals, we still should finish FY-1955 at least $\$ 100,000$ under the Financial Plan.

I

Expenditures for FY-1355 will total about $\$ 8,400,000$. Our Financial Plan request for FY-1956 will total about $\$ 9,400,000$, which is equivalent to about $\$ 785,000$ per month. It currentiy appears that this level will be adequate for our needs. Expenditures for each of several recent months approached this level, but in each case expenditures were inflated by charges for bullding the fluid pressure bonding facility.

\section{DISCLAIMER}

This report was prepared as an account of work sponsored by an agency of the United States States Government nor any agency thereof, nor any of their Government. Neither the United States Go implied, or assumes any legal liability or responsiemployees, makes any warranty, express or implied, of any information, apparatus, product, or bility for the accuracy, completeness, or usefulness of an infringe privately owned rights. Referprocess disclosed, or represents that its use would nocess, or service by trade name, trademark, ence herein to any specific commercial product, procestitute or imply its endorsement, recommanufacturer, or otherwise does not necessarily constiment or any agency thereof. The views mendation, or favoring by the United States Goven necessarily state or reflect those of the

and opinions of authors expressed herein do not 


\section{$\longrightarrow$ \\ APPENDIX B \\ SAVANNAH RTVER LABORATORY \\ PILE MATERIALS PROGRAM \\ JULY 1 TO SEPTEMBER 30, 1955}

DPW-55-15-7

The detalled program for the next three months is described in document DPST-55-320. The goals and the distribution of efforts are as follows:

\begin{tabular}{|c|c|c|c|}
\hline Program & $\begin{array}{c}\text { Apr11 } 1 \\
\text { June } 30 \\
\end{array}$ & $\begin{array}{l}\text { July } 1- \\
\text { Sept. } 30 \\
\end{array}$ & Work to be Completed \\
\hline O slugs & 3,8 & $5 \%$ & $\begin{array}{l}\text {-TA's for Irradiation of } \alpha \text {-Rolled } \\
\text { Slugs. } \\
\text {-Special } \beta \text {-Transformed Slugs. } \\
\text {-Examination of Plmpled Slugs. }\end{array}$ \\
\hline O Plates & $44 \%$ & $55 \%$ & $\begin{array}{l}\text { - Begin Irradiation at SRP. } \\
\text { - Post-Irradiation Examination of } \\
\text { MTR Plates - to be } 50 \% \text { complete at } \\
\text { the end of the period. } \\
\text { - Installation of Non-Bond and Fluoro- } \\
\text { scope Tests. }\end{array}$ \\
\hline O Tubes & $5 \%$ & $17 \%$ & $\begin{array}{l}\text {-Begin Irradiation at MIR. } \\
\text { - Procurement of Al. } \\
\text { - Nickel Plating. } \\
\text {-Fluid-Pressure Bond } 300 \text { Feet of } \\
\text { Tube }\end{array}$ \\
\hline U-AI Slugs & $4 \%$ & $2 \%$ & 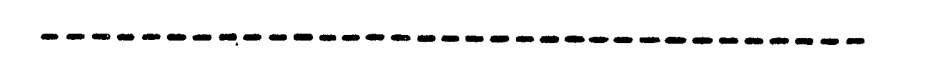 \\
\hline U-AI Tubes & $31 \%$ & $13 \%$ & $\begin{array}{l}\text {-Begin Irradiation at SRP. } \\
\text {-Experimental Tubes. } \\
\text { - } 64 \text { SE Tubes. } \\
\text {-30 SRP Tubes. }\end{array}$ \\
\hline Thor lum & $13 \%$ & $7 \%$ & $\begin{array}{l}\text {-Begin Irradiation of Mark IV Slugs } \\
\text { at SRP. } \\
\text {-Corrosion of Stainless Steel by } \\
\text { Thorex Solutions. }\end{array}$ \\
\hline \multirow{2}{*}{$\begin{array}{l}\text { Oxide Ele- } \\
\text { ments }\end{array}$} & - & $1 \%$ & 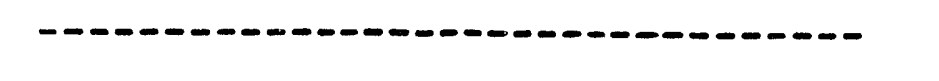 \\
\hline & & & \\
\hline
\end{tabular}


APPENDIX C

SAVANNAH RTVER LABORATORY

INSTRUMENT DEVELOPMENT PROGRAM

JULY 1 TO SEPTEMBER 30, 1955

Study

Code

Name

8508 Instrument Development - 300 Area

Est Imated

Man Months

27

Job \#10-7 Porthole Extrusion Tester

18-1 Flat Plate Non-Bond Tester

18-6 Transformation by Resonance

18-9 LMP Slug Non-Bond Tester

18-10 High Speed Non-Bond Tester

24 NDT Evaluation

8515 Instrument Development - 100 Area

Job \#3-1 Fission Break Monitors

12-2 Matrix Mon1tor

12-3 Three-Dimensional Recorder

26 Poison Computer

28 Wire Flux Monitor

$42 \quad 100$ Area Evaluation

46 Compensated Ion Chamber

56 Automatic Pile Control

57 Flow Monitor

62 Temperature Monitor

8514 Instrument Development - 200 Area

\begin{tabular}{|c|c|}
\hline $\begin{array}{c}\text { Job } \# 1 \\
2-2 \\
5 \\
5-1 \\
5-2 \\
7-2 \\
7-3 \\
8-2 \\
8-3 \\
44 \\
58 \\
69 \\
70 \\
73 \\
76 \\
77 \\
80 \\
81 \\
83 \\
84 \\
85\end{array}$ & 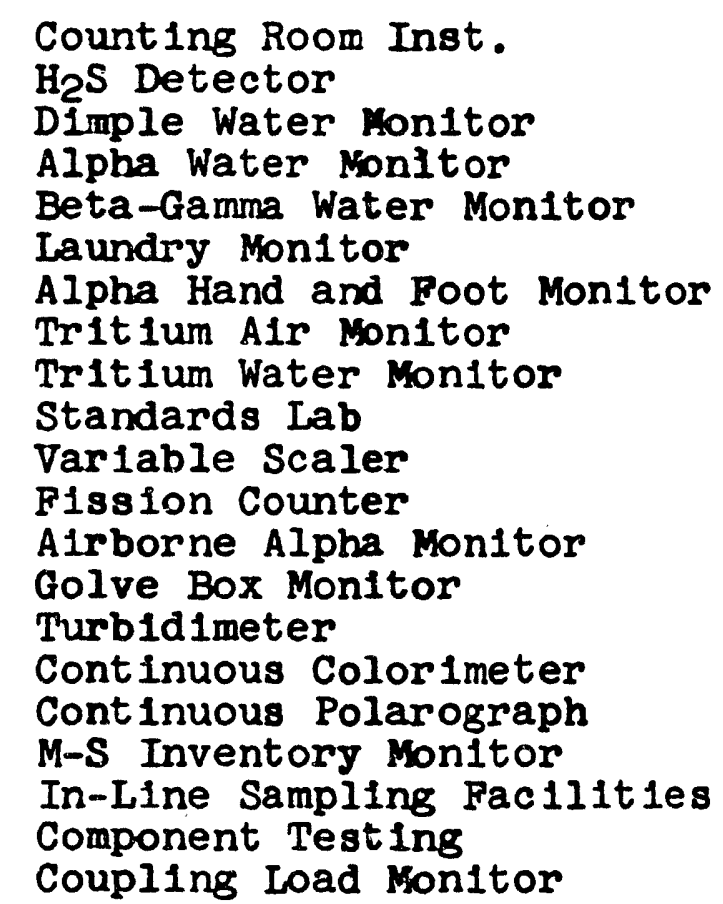 \\
\hline
\end{tabular}




\begin{tabular}{|c|c|c|c|c|c|c|c|c|}
\hline $\begin{array}{l}\text { Study } \\
\text { No. }\end{array}$ & Title & $\begin{array}{l}\text { Man } \\
\text { Mo. }\end{array}$ & From & To & $\begin{array}{c}\text { Man Mo. } \\
\text { Used } \\
\text { Against } \\
\text { Author1z. }\end{array}$ & $\begin{array}{l}\text { Total Man } \\
\text { Mo. Used } \\
\text { FY-1955 } \\
\end{array}$ & Program & Budget \\
\hline 8501 & Non-destruct1ve Testing & 0 & $7 / 1 / 55$ & $9 / 30 / 55$ & 0 & 75.35 & $\begin{array}{l}60 \% \mathrm{MD} \\
40 \% \mathrm{MD}\end{array}$ & $\begin{array}{l}2722 \\
2771\end{array}$ \\
\hline 8502 & $\begin{array}{l}300 \text { Area Process Development - } \\
\text { Present Components }\end{array}$ & 6 & $7 / 1 / 55$ & $9 / 30 / 55$ & 0 & 9.85 & $\mathrm{MD}$ & 2722 \\
\hline 8503 & New Fuel Element Fabrication & 38 & $7 / 1 / 55$ & $9 / 30 / 55$ & 0 & 40 & $\mathrm{MD}$ & 2722 \\
\hline 8504 & $\begin{array}{l}\text { Protective Coatings for New } \\
\text { Fuel Elements }\end{array}$ & $39^{\circ}$ & $7 / 1 / 55$ & $9 / 30 / 55$ & 0 & 97.90 & $M D$ & 2722 \\
\hline 8505 & Corrosion & 13 & $7 / 1 / 55$ & $9 / 30 / 55$ & 0 & 44.90 & $\begin{array}{ll}50 \% & \mathrm{MD} \\
35 \% & \mathrm{MD} \\
15 \% & \mathrm{MD}\end{array}$ & $\begin{array}{l}2722 \\
2742 \\
2771\end{array}$ \\
\hline 8506 & $\begin{array}{l}100 \text { Area Process Development - } \\
\text { General }\end{array}$ & 15 & $5 / 1 / 55$ & $7 / 31 / 55$ & 7.65 & 54.75 & RPD & 2723 \\
\hline 8508 & $\begin{array}{l}\text { Instrument Development - } 300 \\
\text { Area }\end{array}$ & 27 & $7 / 1 / 55$ & $9 / 30 / 55$ & 0 & 40 & $\begin{array}{l}75 \% \mathrm{MD} \\
10 \% \\
15 \% \mathrm{MD}\end{array}$ & $\begin{array}{l}2722 \\
2742 \\
2771\end{array}$ \\
\hline 8509 & $\begin{array}{l}\text { Design and Evaluation of Fuel } \\
\text { Elements }\end{array}$ & 58.5 & $5 / 1 / 55$ & $7 / 31 / 55$ & 33.85 & 178.95 & $\begin{array}{l}\text { 60\% RPD } \\
40 \% \text { RPD }\end{array}$ & $\begin{array}{l}2723 \\
2743\end{array}$ \\
\hline 8511 & $\begin{array}{l}\text { Separations Process and Equip- } \\
\text { ment Demonstration }\end{array}$ & 45 & $6 / 1 / 55$ & $8 / 31 / 55$ & 13.05 & 132.95 & $\begin{array}{rl}95 \% & \mathrm{CPD} \\
5 \% & \mathrm{CPD}\end{array}$ & $\begin{array}{l}2724 \\
2730\end{array}$ \\
\hline 8512 & Separations Process Chemistry & 58 & $6 / 1 / 55$ & $8 / 31 / 55$ & 15.55 & 182.45 & $\begin{array}{l}\text { 95\% CPD } \\
5 \% \text { CPD }\end{array}$ & $\begin{array}{l}2724 \\
2744\end{array}$ \\
\hline 8513 & $\begin{array}{l}\text { Separations Engineering De- } \\
\text { velopment }\end{array}$ & 45 & $6 / 1 / 55$ & $8 / 31 / 55$ & 11.90 & 135.85 & $\begin{array}{r}90 \% \text { CPD } \\
5 \% \text { CPD } \\
5 \% \text { CPD }\end{array}$ & $\begin{array}{l}2724 \\
2730 \\
2744\end{array}$ \\
\hline \multicolumn{8}{|c|}{ UNCLASS IF IED } & Page 6 \\
\hline
\end{tabular}




\begin{tabular}{|c|c|c|c|c|c|c|c|c|}
\hline $\begin{array}{l}\text { Study } \\
\text { No. }\end{array}$ & $\mathrm{T} 1 \mathrm{t} 1 \mathrm{e}$ & $\begin{array}{l}\text { Man } \\
\text { Mo..-. }\end{array}$ & From & To & $\begin{array}{l}\text { Man Mo. } \\
\text { Used } \\
\text { Aga1nst } \\
\text { Author 1z. }\end{array}$ & $\begin{array}{l}\text { Total Man } \\
\text { Mo. Used } \\
\text { FY - } 1955 \\
\end{array}$ & Program & Budget \\
\hline 8514 & $\begin{array}{l}\text { Instrument Development - } 200 \\
\text { Area }\end{array}$ & 42 & $7 / 1 / 55$ & $9 / 30 / 55$ & 0 & 54.20 & $\begin{array}{rl}85 \% & C P D \\
10 \% & C P D \\
5 \% & C P D\end{array}$ & $\begin{array}{l}2724 \\
2730 \\
2760\end{array}$ \\
\hline 8515 & $\begin{array}{l}\text { Instrument Development - } 100 \\
\text { Area }\end{array}$ & 15 & $7 / 1 / 55$ & $9 / 30 / 55$ & 0 & 35.70 & $\begin{array}{rl}90 \% & R P D \\
5 \% & R P D \\
5 \% & R P D\end{array}$ & $\begin{array}{l}2723 \\
2730 \\
2743\end{array}$ \\
\hline 8517 & Separations Process Hazards & 3 & $6 / 1 / 55$ & $8 / 31 / 55$ & .20 & 12 & CPD & 2724 \\
\hline 8518 & Theoretical Physics & 40 & $5 / 1 / 55$ & $7 / 31 / 55$ & 20.25 & 120.75 & $\begin{array}{rl}75 \% & R P D \\
5 \% & R P D \\
20 \% & R P D\end{array}$ & $\begin{array}{l}2723 \\
2730 \\
2743\end{array}$ \\
\hline $85 \geq 9$ & Experimental P1le Physics & 68 & $5 / 1 / 55$ & $7 / 31 / 55$ & 40.50 & 254.85 & $\begin{array}{ll}50 \% & R P D \\
10 \% & R P D \\
40 \% & R P D\end{array}$ & $\begin{array}{l}2723 \\
2730 \\
2743\end{array}$ \\
\hline 8520 & $\begin{array}{l}100 \text { Area Mechanical Develop- } \\
\text { mont }\end{array}$ & 40.5 & $5 / 1 / 55$ & $7 / 31 / 55$ & 24.40 & 141.60 & $\begin{array}{l}\text { 65\% RPD } \\
35 \% \text { RPD }\end{array}$ & $\begin{array}{l}2723 \\
2743\end{array}$ \\
\hline 8521 & Hydriside Development & 2 & $6 / 1 / 55$ & $8 / 31 / 55$ & 1.15 & 24.10 & CPD & 2730 \\
\hline 8522 & $\begin{array}{l}\text { Analytical Chemistry } \\
\text { Development }\end{array}$ & 29 & $6 / 1 / 55$ & $8 / 31 / 55$ & 7.45 & 102.90 & $\begin{array}{l}10 \% \mathrm{RPD} \\
70 \% \mathrm{CPD} \\
20 \% \mathrm{MD}\end{array}$ & $\begin{array}{l}2723 \\
2724 \\
2742\end{array}$ \\
\hline 8523 & Waste Handling & 3 & $6 / 1 / 55$ & $8 / 31 / 55$ & .45 & 3.30 & $\mathrm{CPD}$ & 2724 \\
\hline 8524 & New LM Elements & 18 & $7 / 1 / 55$ & $9 / 30 / 55$ & 0 & 147.55 & $\begin{array}{ll}20 \% & M D \\
80 \% & M D\end{array}$ & $\begin{array}{l}2742 \\
2771\end{array}$ \\
\hline $\begin{array}{l}8525 \\
8526\end{array}$ & $\begin{array}{l}\text { Fluid Pressure Facility } \\
\text { Recycle Fac1l1ty }\end{array}$ & & $\begin{array}{l}\text { (For } \\
\text { (For }\end{array}$ & $\begin{array}{l}\text { construct } \\
\text { construct }\end{array}$ & $\begin{array}{l}\text { tion only) } \\
\text { tion only) }\end{array}$ & & CPD & 2722 \\
\hline $\begin{array}{l}8527 \\
8528\end{array}$ & Recycle Development & 6 & $6 / 1 / 55$ & $8 / 31 / 55$ & 2.10 & 10.95 & CPD & 2704 \\
\hline \multirow{2}{*}{8528} & Heat Treating Fac1lity & & (For & construct & tion only) & & MD & 2722 \\
\hline & & & UNCLASS IF & IED & & & & Page 7 \\
\hline
\end{tabular}



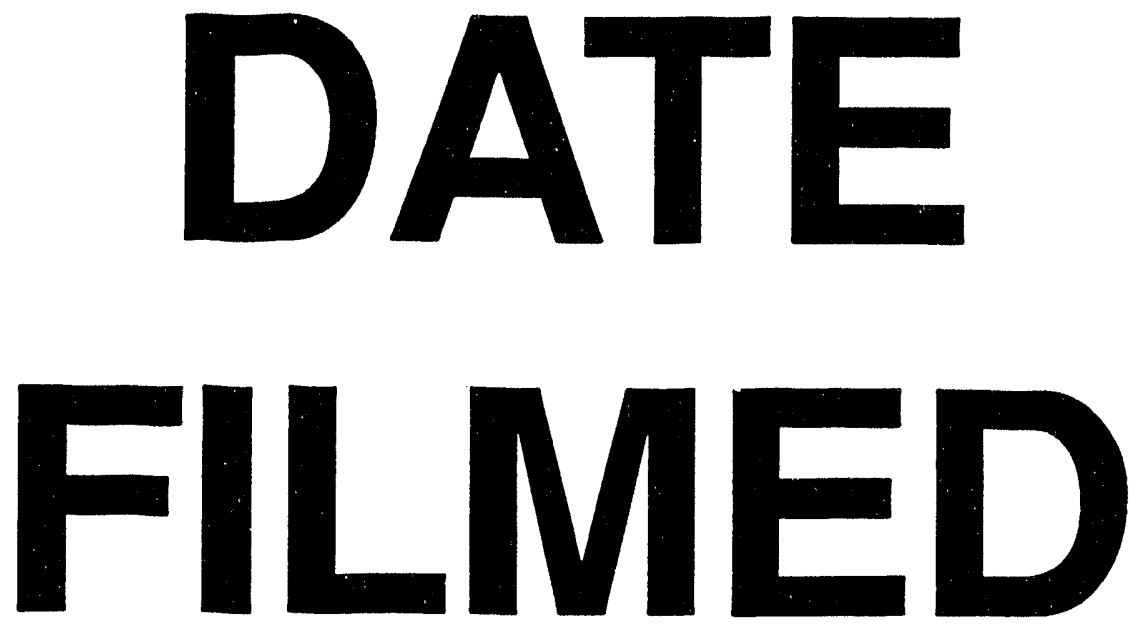

$10 / 28 / 94$
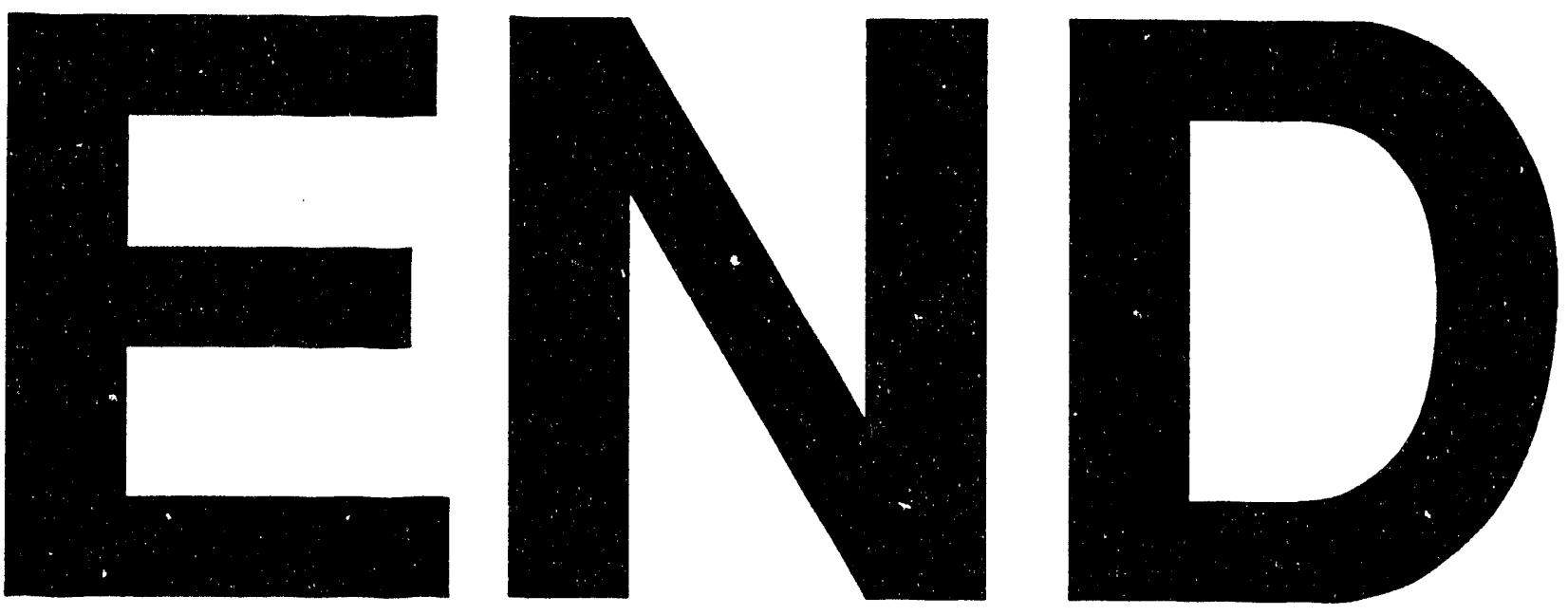
